\title{
Design of an Interaction Region for the Linac-Ring Version of the Electron-Ion Collider ERHIC
}

C. Montag, J. Kewisch, I. Ben-Zvi

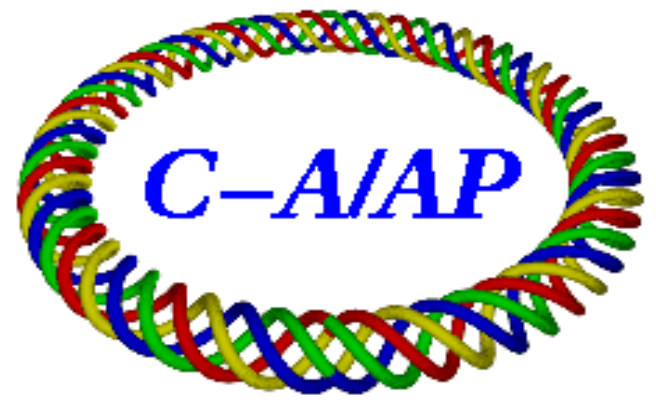

Collider-Accelerator Department Brookhaven National Laboratory Upton, NY 11973 


\title{
DESIGN OF AN INTERACTION REGION FOR THE LINAC-RING VERSION OF THE ELECTRON-ION COLLIDER ERHIC*
}

\author{
C. Montag $\jmath^{\dagger}$ J. Kewisch, I. Ben-Zvi
}

January 21, 2004

\begin{abstract}
An interaction region design for the linac-ring version of eRHIC is presented which provides an e-p luminosity of $9 \cdot 10^{32} \mathrm{~cm}^{-2} \mathrm{sec}^{-1}$ for a RHIC proton bunch intensity of $N_{p}=1.0 \cdot 10^{11}$ and a proton beam-beam tuneshift of $\xi_{p}=0.0048$.
\end{abstract}

\section{Introduction}

Two approaches are currently being considered for the electron-ion collider eR$\mathrm{HIC}$, consisting of one of the existing RHIC storage rings and a $10 \mathrm{GeV}$ electron accelerator. One approach utilizes a $10 \mathrm{GeV}$ electron storage ring, while the other aims at using a $10 \mathrm{GeV}$ superconducting energy-recovery linac. Table 1 summarizes some basic parameters of the linac-ring version of eRHIC.

The interaction region of this machine has to serve several purposes. Both beams have to be focussed by low- $\beta$ quadrupole magnets to obtain small beam sizes at the interaction point (IP), thus maximizing the luminosity. Secondly, the beams have to be separated as close to the IP as possible to allow for the low$\beta$ quadrupoles to be installed close to the IP. With beam energies differing by more than an order of magnitude, both beams can share the low- $\beta$ quadrupoles of the electron beam, which have practically no effect on the high-energy hadron beam. These magnets are installed inside the detector volume. To minimize the actual detector volume occupied by these magnets, superconducting magnets similar to the HERA luminosity upgrade design [1] are foreseen. These magnets are equipped with an additional dipole coil to provide the required beam separation, deflecting the electron beam while leaving the higher-energy hadron beam orbit practically unchanged.

*Work performed under contract number DE-AC02-98CH10886 with the auspices of the US Department of Energy

†montag@bnl.gov 


\begin{tabular}{lr}
\hline \hline electrons: & \\
beam energy [GeV] & 10 \\
geometric emittance hor./vert. [nm] & $2.5 / 2.5$ \\
$\beta$ functions hor./vert. [m] & $1.0 / 1.0$ \\
particles/bunch & $1.0 \cdot 10^{11}$ \\
\hline hadrons: & 3834 \\
ring circumference [m] & $250(\mathrm{p})$ \\
beam energy [GeV] & $9.5 / 9.5$ \\
geometric emittance hor./vert. [nm] & $0.26 / 0.26$ \\
$\beta$ functions hor./vert. [m] & $1.0 \cdot 10^{11}(\mathrm{p})$ \\
particles/bunch & 360 \\
number of bunches & $0.0048 / 0.0048$ \\
beam-beam tune shift hor./vert. & $0.9 \cdot 10^{33}$ \\
\hline luminosity [cm $\left.\mathrm{cm}^{-2} \mathrm{sec}^{-1}\right]$ &
\end{tabular}

Table 1: Parameters of the linac-ring version of eRHIC.

With this scheme, the first hadron low- $\beta$ quadrupole, Q1, is installed at a distance of $5 \mathrm{~m}$ from the IP (see Figure 1). This requires the electrons to be deflected sufficiently to be guided through a field-free region outside this magnet. Since this separation generates synchrotron radiation, it is mandatory to keep it as small as possible. This is achieved by installation of septum quadrupoles for the hadron beam.

This report documents the current status of the interaction region design of

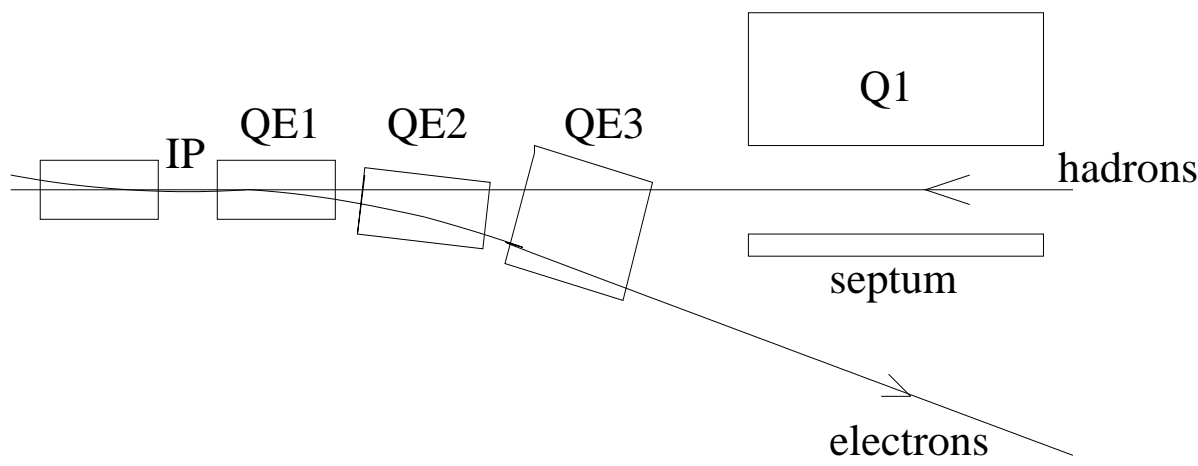

Figure 1: Schematic view of the eRHIC interaction region.

the linac-ring version of eRHIC. 


\section{Hadron IR optics}

Low- $\beta$ focussing of the hadron beam to $\beta_{x}=\beta_{y}=0.26 \mathrm{~m}$ is provided by a normal-conducting quadrupole triplet. The use of septum-quadrupoles for all these magnets minimizes the required beam separation between electrons and hadrons, and provides maximum freedom for the installation of magnets in the electron beam line.

The first and second lens of the triplet are split up into various individual magnets, with pole tip radii tailored to the varying beam size. This ensures a sufficient aperture of $12 \sigma_{p}$, while simultaneously minimizing the total length of the low- $\beta$ system. The resulting $\beta$ functions and magnet positions are shown in Figure 2.

Pole tip fields are limited to $1.0 \mathrm{~T}$ to avoid field quality degradation due to saturation effects. Table 2 lists the resulting magnet parameters, while cross sections of the entrance and exit of each magnet with its pole tip radius and the $12 \sigma$ beam ellipse are shown in Figures 3 to 11 . In these figures, space for the vacuum chamber is accounted for by additional lines parallel to the septum plate and the magnet poles.

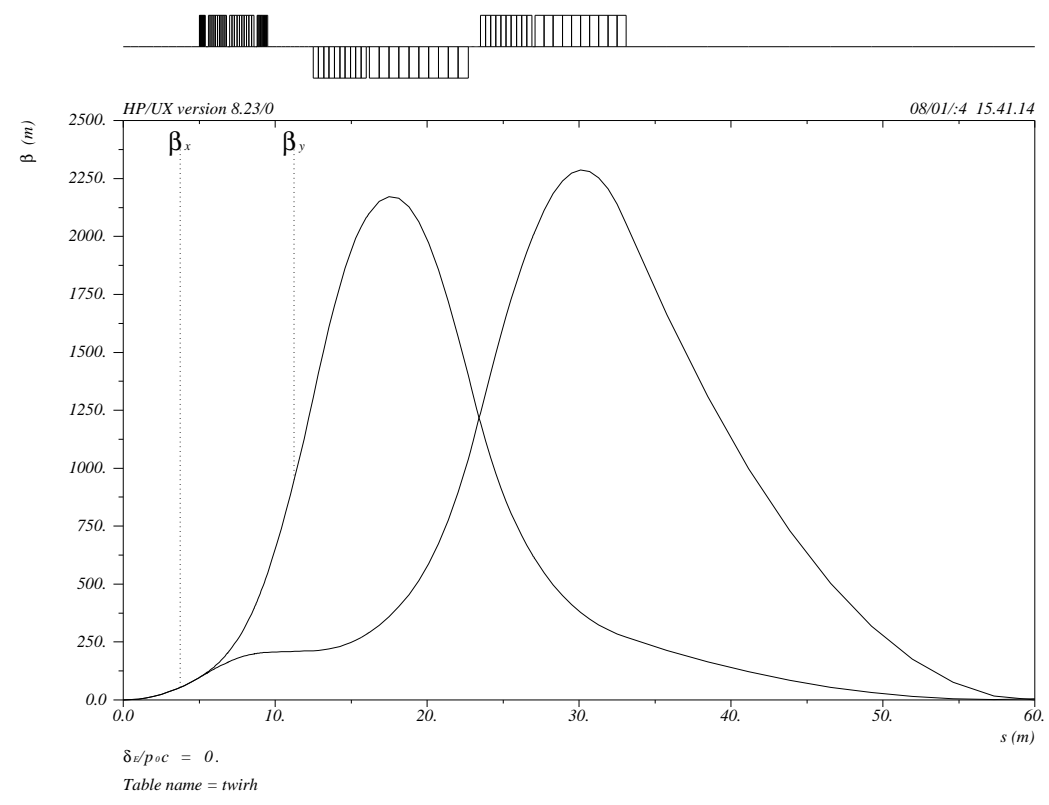

Figure 2: Hadron IR lattice for the electron-ion collider eRHIC. 


\begin{tabular}{lr}
\hline Q1: & \\
length [m] & 0.4 \\
gradient [T/m] & 43.5 \\
pole tip radius [mm] & 23.0 \\
pole tip field [T] & 1.0 \\
\hline Q1B: & \\
length [m] & 1.2 \\
gradient [T/m] & 35.7 \\
pole tip radius [mm] & 28.0 \\
pole tip field [T] & 1.0 \\
\hline Q1C: & \\
length [m] & 1.6 \\
gradient [T/m] & 29.2 \\
pole tip radius [mm] & 34.3 \\
pole tip field [T] & 1.0 \\
\hline Q1D: & \\
length [m] & 0.7 \\
gradient [T/m] & 25.0 \\
pole tip radius [mm] & 40.0 \\
pole tip field [T] & 1.0 \\
\hline \hline Q2: & \\
length [m] & 3.5 \\
gradient [T/m] & 18.0 \\
pole tip radius [mm] & 55.6 \\
pole tip field [T] & 1.0 \\
\hline Q2B: & \\
length [m] & 6.5 \\
gradient [T/m] & 13.3 \\
pole tip radius [mm] & 75.0 \\
pole tip field [T] & 1.0 \\
\hline \hline Q3: & \\
length [m] & \\
gradient [T/m] & 11.3 \\
pole tip radius [mm] & 88.9 \\
pole tip field [T] & 1.0 \\
\hline Q3B: & \\
length [m] \\
gradient [T/m] & 10.4 \\
pole tip radius [mm] & 96.0 \\
pole tip field [T] & 1.0 \\
\hline
\end{tabular}

Table 2: Parameter list of the hadron low- $\beta$ septum quadrupoles. 


\section{$3 \quad$ Electron IR optics}

As shown in Table 1 , the electron beam is focussed to $\beta_{x}=\beta_{y}=1.0 \mathrm{~m}$ at the IP. This rather large $\beta$ function is easy to obtain, and does not necessarily require focussing close to the IP. Figure 12 shows a possible low- $\beta$ focussing, using a quadrupole triplet. This scheme leaves an element free space of $\pm 1.0 \mathrm{~m}$ around the IP, which can be easily expanded if necessary. To minimize the required separation at the septum, the horizontal $\beta$ function at the $5 \mathrm{~m}$ septum location has been minimized.

With the very small electron beam emittance of $2.5 \mathrm{~nm}$ and a maximum electron beta function of only $20 \mathrm{~m}$ inside the triplet, the required beam pipe radius is determined by the width of the synchrotron radiation fan generated by the separation dipole coils. With a pole tip field of $2.0 \mathrm{~T}$, a sufficient aperture is achieved. Table 3 lists some basic magnet parameters of the superconducting quadrupole triplet.

Matching into the recirculating linac can be provided by normal-conducting septum quadrupole magnets. These magnets can be placed anywhere as soon as the separation between the two beam lines (electrons and hadrons) is large enough to allow for an electron and a hadron septum quadrupole to be placed side-by-side. This is the case for distances larger than some $10 \mathrm{~m}$ from the IP. The resulting very moderate $\beta$ functions at $10 \mathrm{~m}$ can easily be focussed into the linac, most likely by a second quadrupole triplet. Additionally, the triplet quadrupoles themselves can be somewhat re-adjusted for matching into the linac.

\section{Synchrotron radiation}

The superconducting electron low- $\beta$ triplet inside the detector is shared by both particle beams as well as the synchrotron radiation fan resulting from dipole windings necessary for beam separation. At the location of the septum, $5 \mathrm{~m}$ from the IP, the required separation is given by the $12 \sigma_{p, x}$ beam size of the hadron beam, plus the thickness $d_{\text {septum }}=10 \mathrm{~mm}$ of the septum itself, plus some sufficient aperture for the electron beam. In contrast to the ring-ring case, where $20 \sigma_{e, x}$ are mandatory for sufficient electron beam lifetime and minimum background conditions, this condition is much more relaxed in the linac-ring approach for two reasons. First of all, an aperture of $5 \ldots 10 \sigma_{e, x}$ is sufficient in the linac-ring approach, where the beam passes through the interaction region only once. Second, the horizontal electron beam emittance is more than an order of magnitude smaller. Using the beam emittances given in Table 1 and the $\beta$ functions shown in Figures 2 and 12, the required separation is therefore computed as

$$
\begin{aligned}
\Delta x & =12 \sigma_{p, x}+10 \sigma_{e, x}+d_{\text {septum }} \\
& =12 \cdot \sqrt{\beta_{x, p} \epsilon_{p}}+10 \cdot \sqrt{\beta_{x, e} \epsilon_{e}}+d_{\text {septum }} \\
& =12 \cdot 0.93 \mathrm{~mm}+10 \cdot 0.08 \mathrm{~mm}+10 \mathrm{~mm}
\end{aligned}
$$




\begin{tabular}{lr}
\hline \hline QE1: & \\
length [m] & 0.6 \\
gradient [T/m] & 83.3 \\
radius [mm] & 24 \\
bending angle left/right [mrad] & $3.7 /-3.0$ \\
shift w.r.t. detector axis left/right [mm] & $0 /-10$ \\
tilt w.r.t. detector axis left/right [mrad] & $1.85 /-1.5$ \\
synchrotron radiation power left/right [W] & $1609 / 1058$ \\
synchr. rad. power on septum left/right [W] & $800 / 0$ \\
critical photon energy left/right [keV] & $13.7 / 11.1$ \\
\hline QE2: & \\
length [m] & 0.8 \\
gradient [T/m] & 76.7 \\
radius [mm] & 26 \\
bending angle left/right [mrad] & $3.8 /-2.4$ \\
shift w.r.t. detextor axis left/right [mm] & $0 /-10$ \\
tilt w.r.t. detector axis left/right [mrad] & $3.75 /-2.7$ \\
synchrotron radiation power left/right [W] & $1273 / 508$ \\
synchr. rad. power on septum left/right [W] & $0 / 508$ \\
critical photon energy left/right [keV] & $10.5 / 6.7$ \\
\hline QE3: & 0.6 \\
length [m] & 56.7 \\
gradient [T/m] & 35 \\
radius [mm] & $0 /-2.4$ \\
bending angle left/right [mrad] & $0 /-10$ \\
shift w.r.t. detector axis left/right [mm] & $3.75 /-3.6$ \\
tilt w.r.t. detector axis left/right [mrad] & $0 / 677$ \\
synchrotron radiation power left/right [kW] & $0 / 340$ \\
synchr. rad. power on septum left/right [kW] & $0 / 8.9$ \\
critical photon energy left/right [keV] & \\
\hline & \\
\hline
\end{tabular}

Table 3: Magnet parameters for the electron triplet. 


$$
=22 \mathrm{~mm} \text {. }
$$

When providing this separation by the dipole windings in the superconducting electron triplet quadrupoles, a synchrotron radiation fan is produced that has to be passed safely through the detector and the superconducting magnets. At the right side of the detector (electron-downstream), some fraction of this fan unavoidably hits the septum of the first hadron quadrupole Q1. To minimize back-scattering of these synchrotron radiation photons into the detector, the total power as well as the critical photon energy of the synchrotron radiation hitting the septum have to be minimized by distributing the required bending angles among the various magnets.

On the right side of the detector, this optimization is accomplished by setting the QE1 dipole to a rather large strength, resulting in a synchrotron radiation fan that just covers the angle between the hadron orbit and the septum. The dipole coils of QE2 and QE3 then share the required remaining separation angle. On the left side, the dipole coil in the QE3 has to be set to zero to minimize the resulting width of the synchrotron radiation fan, especially inside the triplet on the right. Here, the separation is provided by QE1 and QE2 only. Table 3 lists bending angles and synchrotron radiation parameters of these magnets.

To further decrease the required magnet aperture, the triplet quadrupoles QE1, QE2, and QE3 on the right side of the IP are shifted away from the hadron beam orbit by an additional $10 \mathrm{~mm}$. The resulting effect on the electron orbit is compensated by the dipole coils in these magnets. Figure 13 shows the IR geometry with the quadrupole triplets and the synchrotron radiation fan.

\section{Conclusion}

The eRHIC interaction region presented in this paper provides an e-p luminosity of $0.9 \cdot 10^{33} \mathrm{~cm}^{-2} \mathrm{sec}^{-1}$. Since the beam-beam effect on the electron beam can be ignored in the linac-ring approach, this luminosity can be increased by raising the hadron bunch intensity. The beam-beam tune shift of $\xi_{x}=\xi_{y}=0.0048$ for the hadron beam is well within values presently achieved at RHIC, and therefore considered achievable for eRHIC. Minimum apertures of $10 \sigma_{e}$ for the electrons and $12 \sigma_{p}$ for the hadrons are achieved in this design, which is consid-

ered sufficient both for safe operation as well as minimum detector background conditions.

\section{References}

[1] U. Schneekloth (ed.), The HERA Luminosity Upgrade, DESY HERA 98-05 

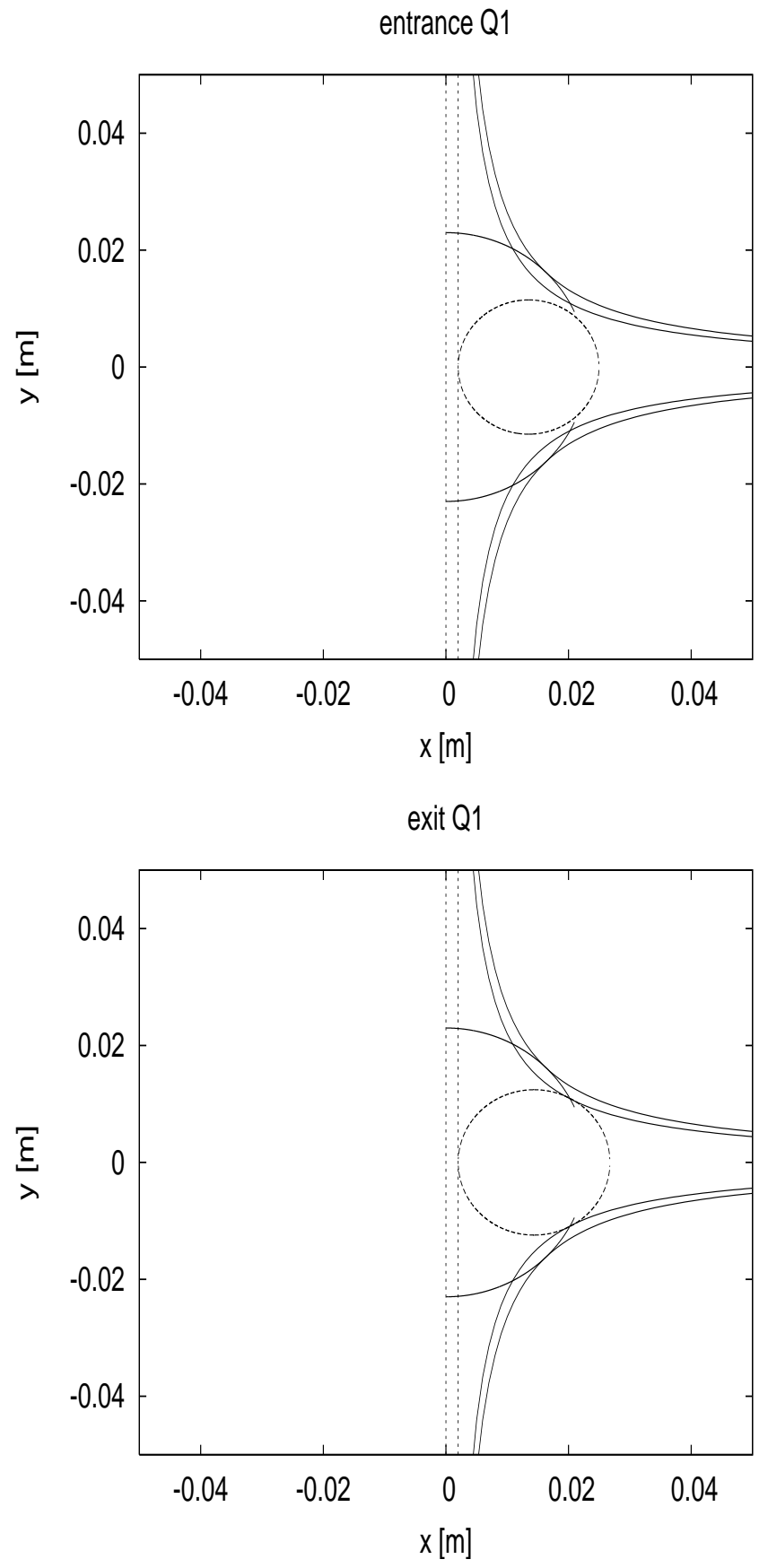

Figure 3: Entrance and exit of the hadron magnet Q1, for a pole tip field of $1.0 \mathrm{~T}$. 

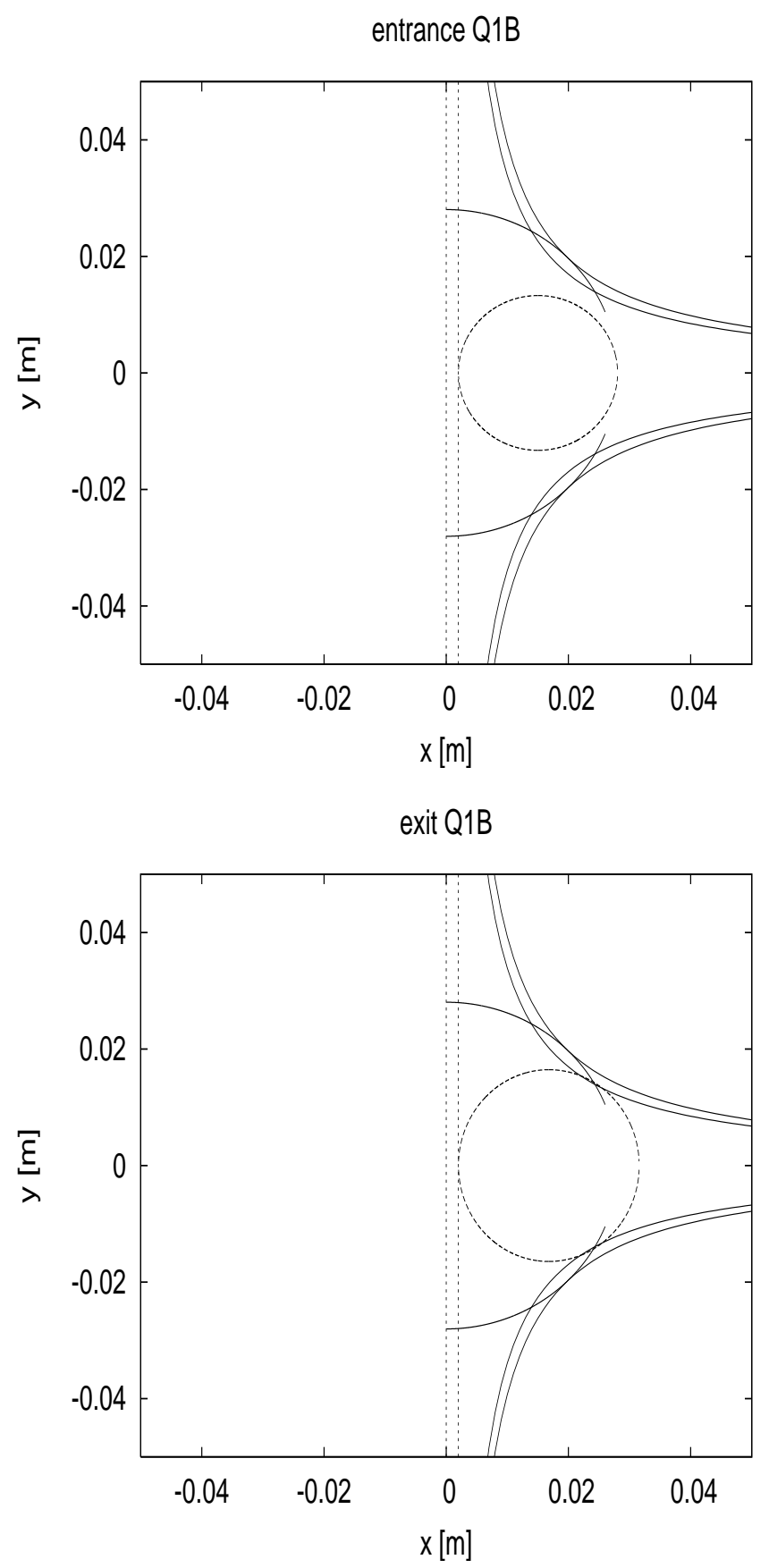

Figure 4: Entrance and exit of the hadron magnet Q1B, for a pole tip field of $1.0 \mathrm{~T}$. 

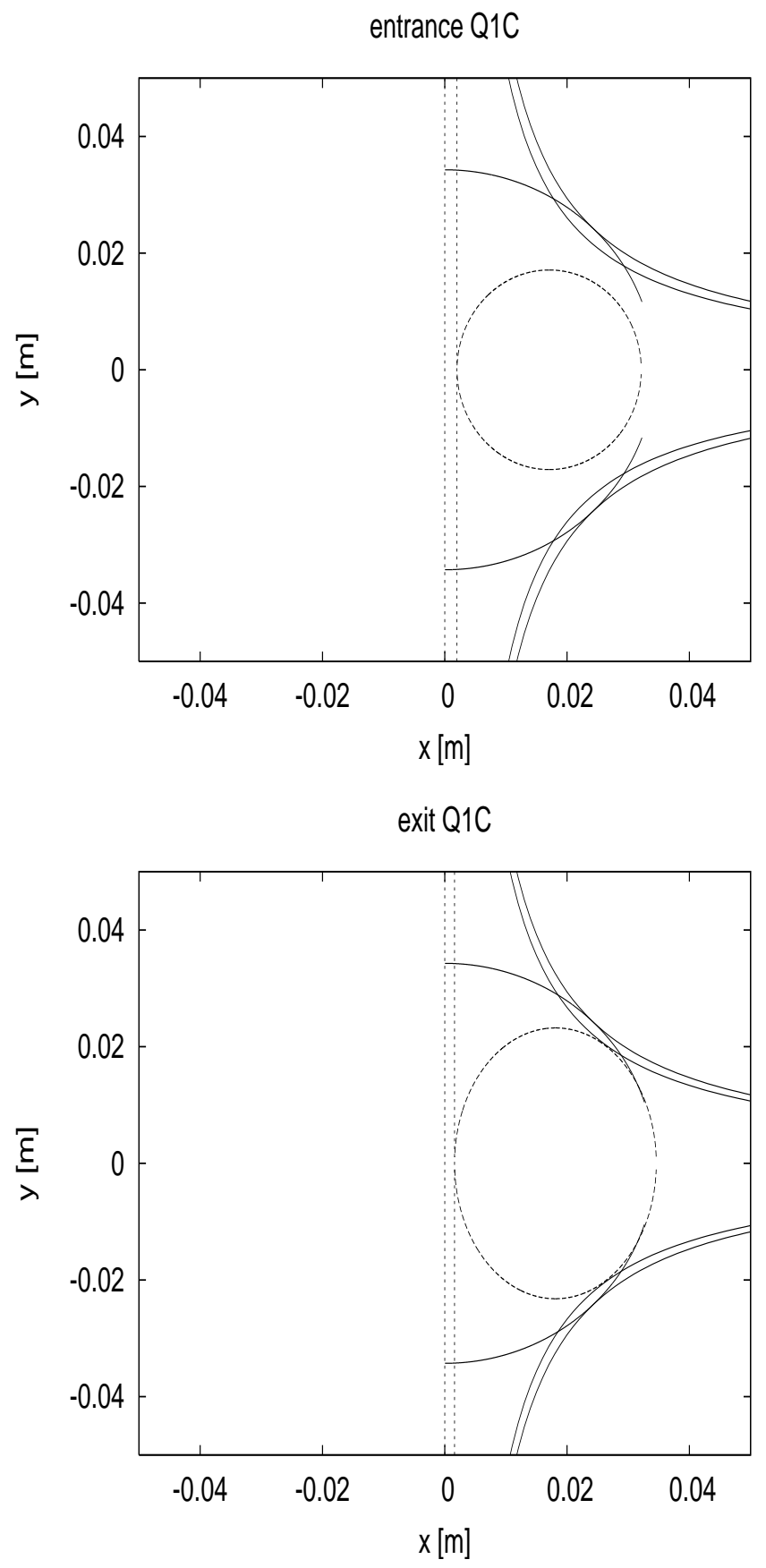

Figure 5: Entrance and exit of the hadron magnet Q1C, for a pole tip field of $1.0 \mathrm{~T}$. 

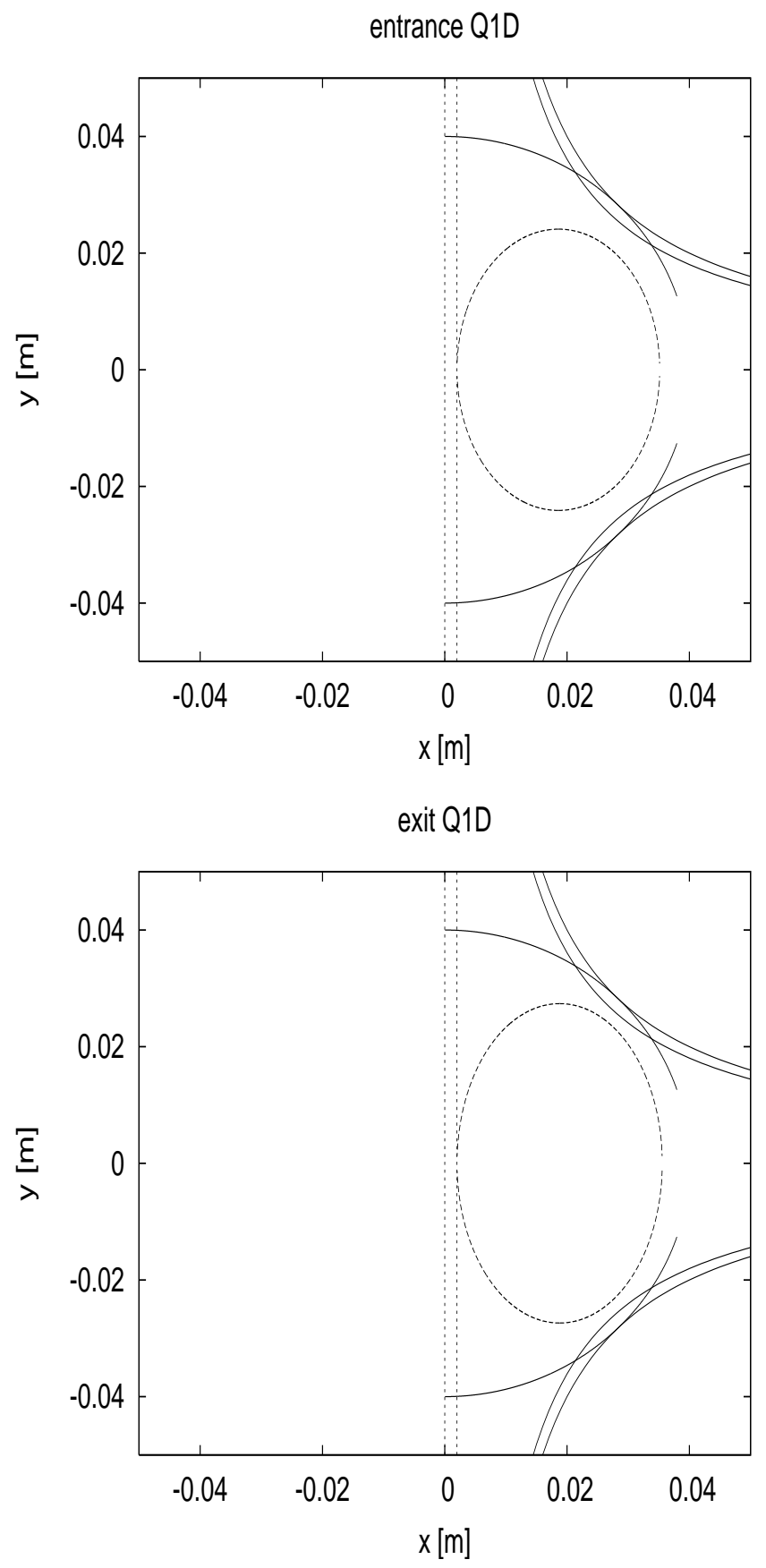

Figure 6: Entrance and exit of the hadron magnet Q1D, for a pole tip field of $1.0 \mathrm{~T}$. 

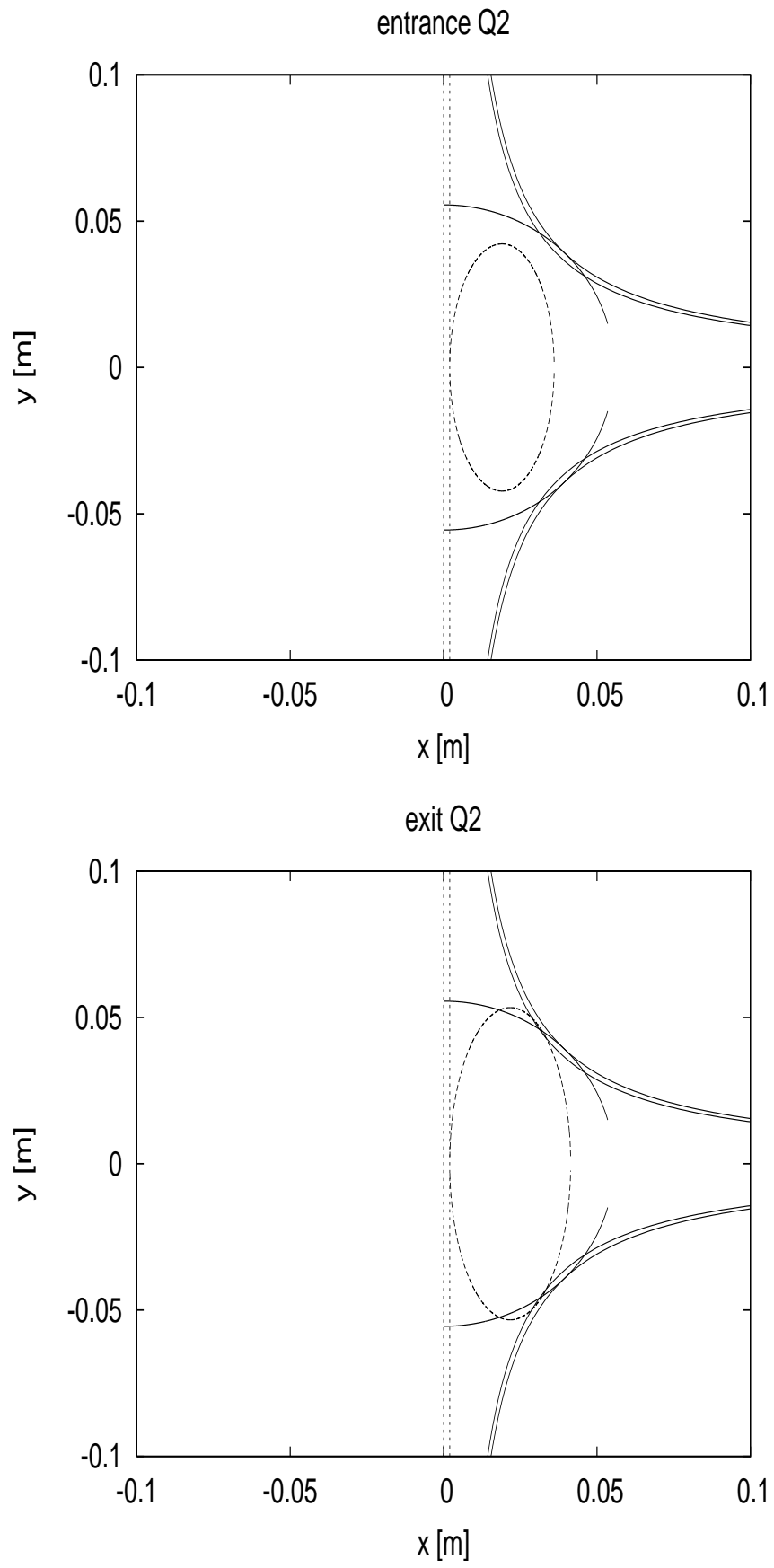

Figure 7: Entrance and exit of the hadron magnet Q2, for a pole tip field of $1.0 \mathrm{~T}$. 

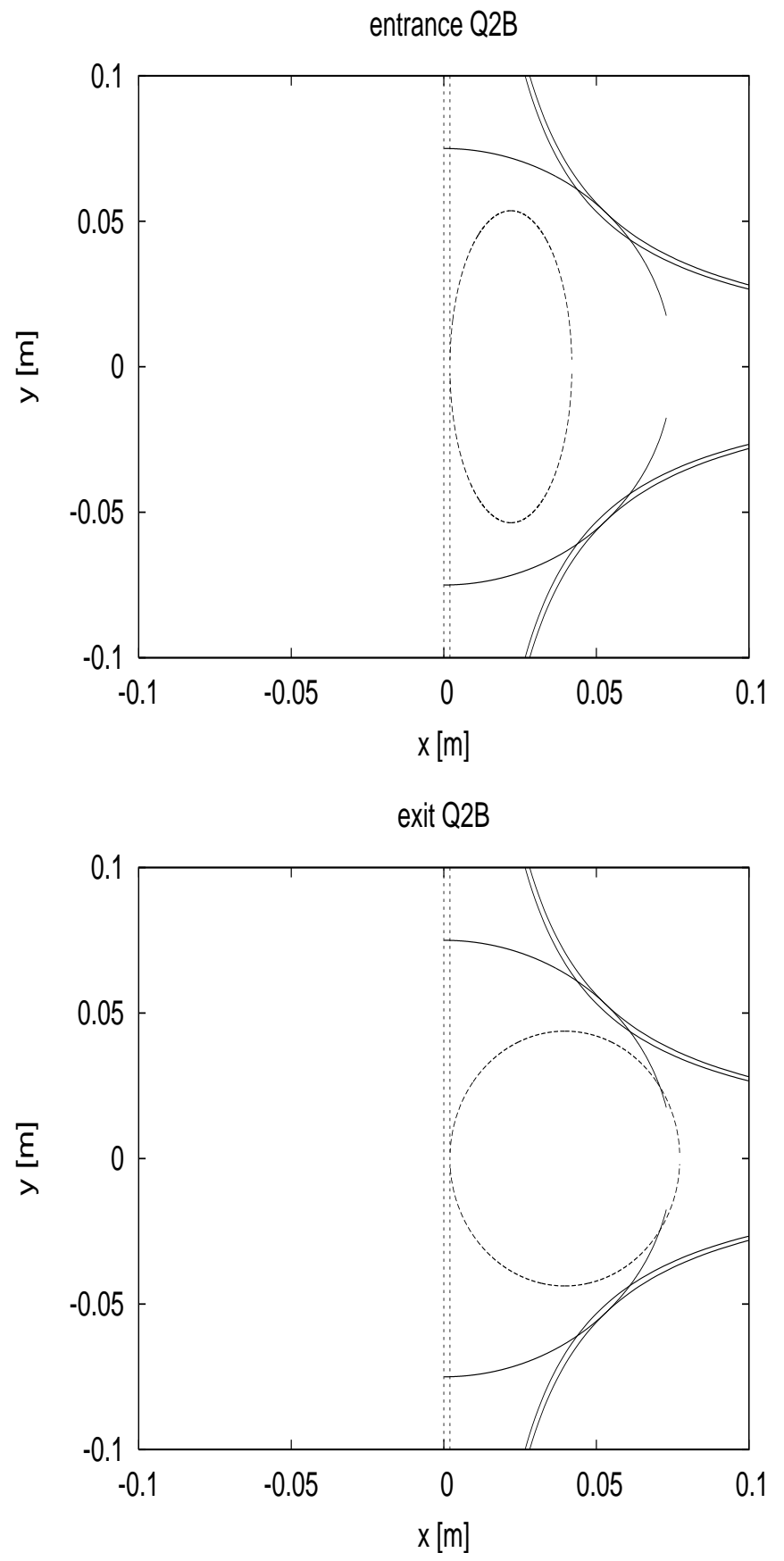

Figure 8: Entrance and exit of the hadron magnet Q2B. 

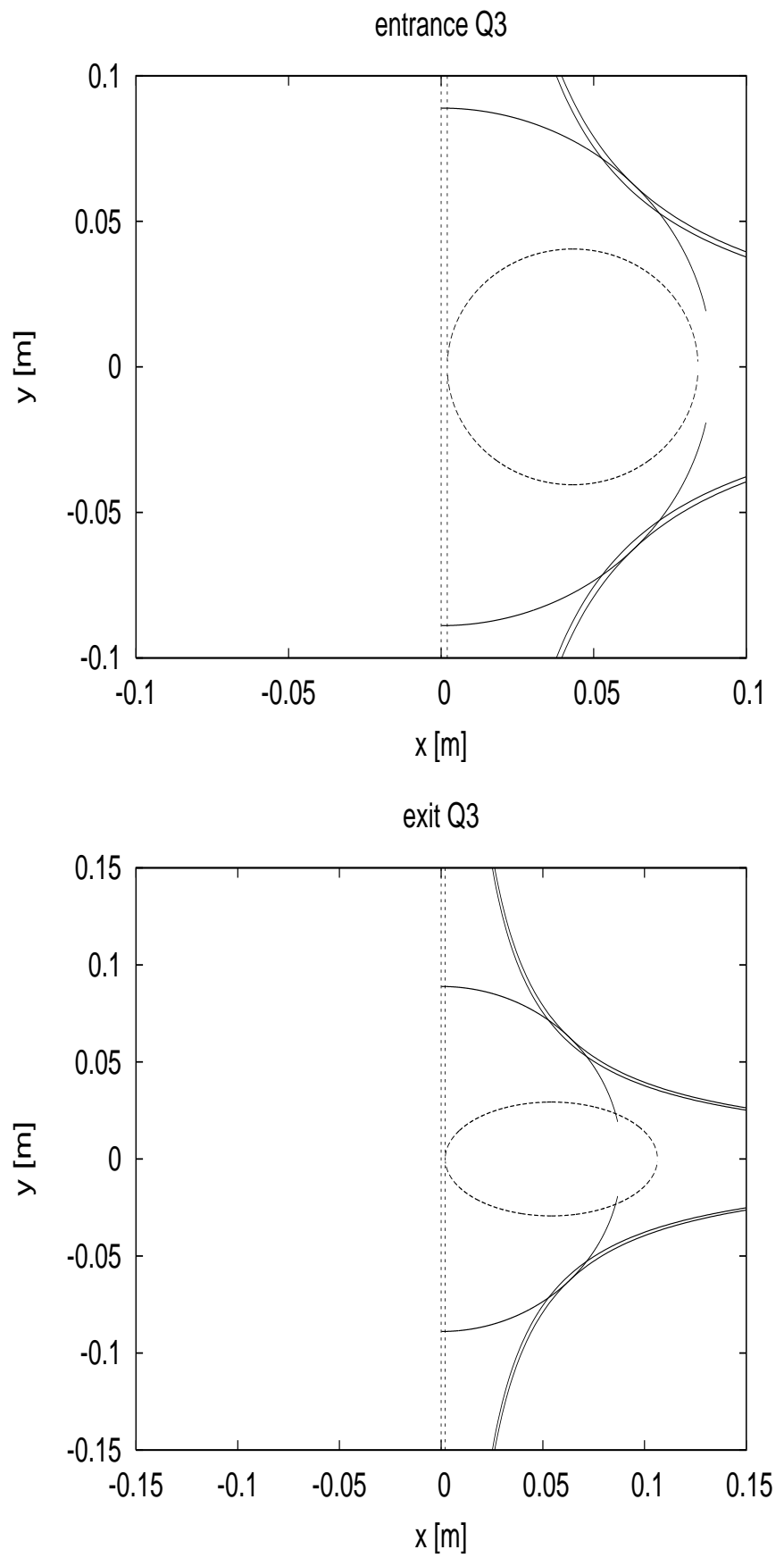

Figure 9: Entrance and exit of the hadron magnet Q3. 

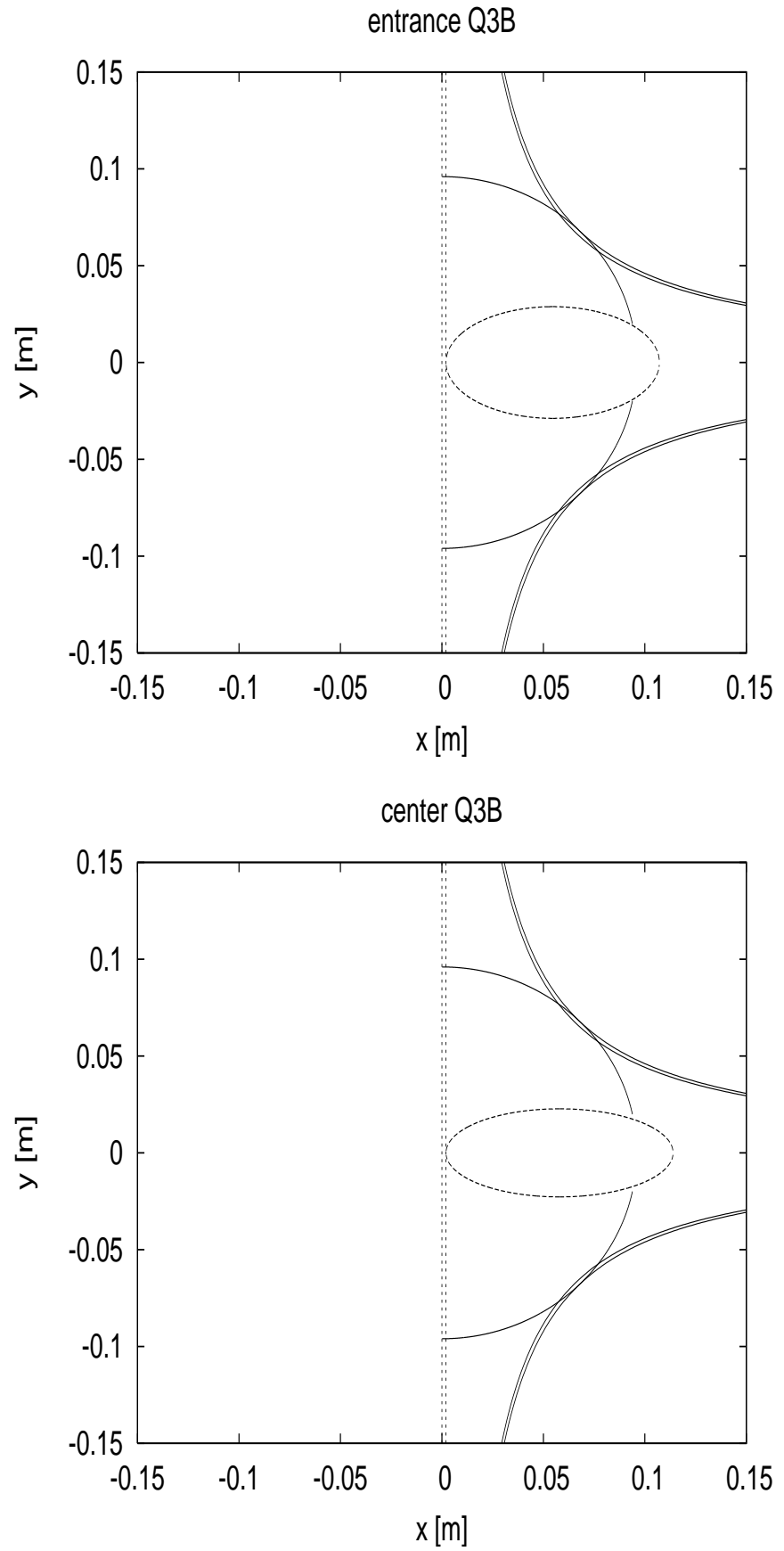

Figure 10: Entrance and center of the hadron magnet Q3. 


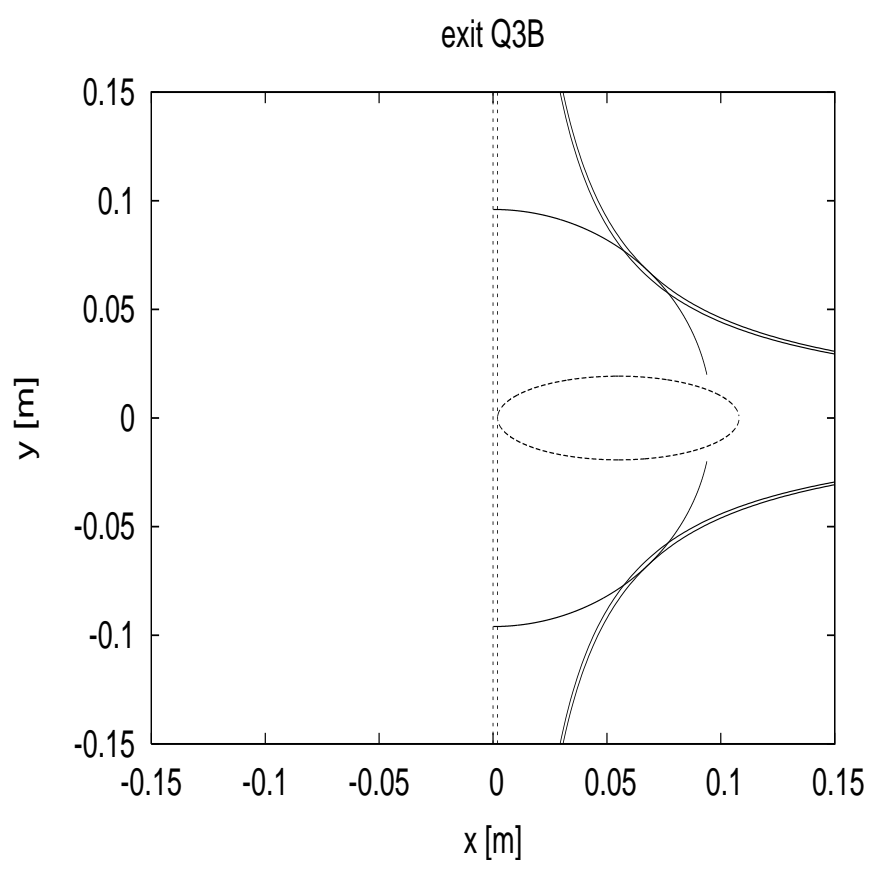

Figure 11: Exit of the hadron magnet Q3. 


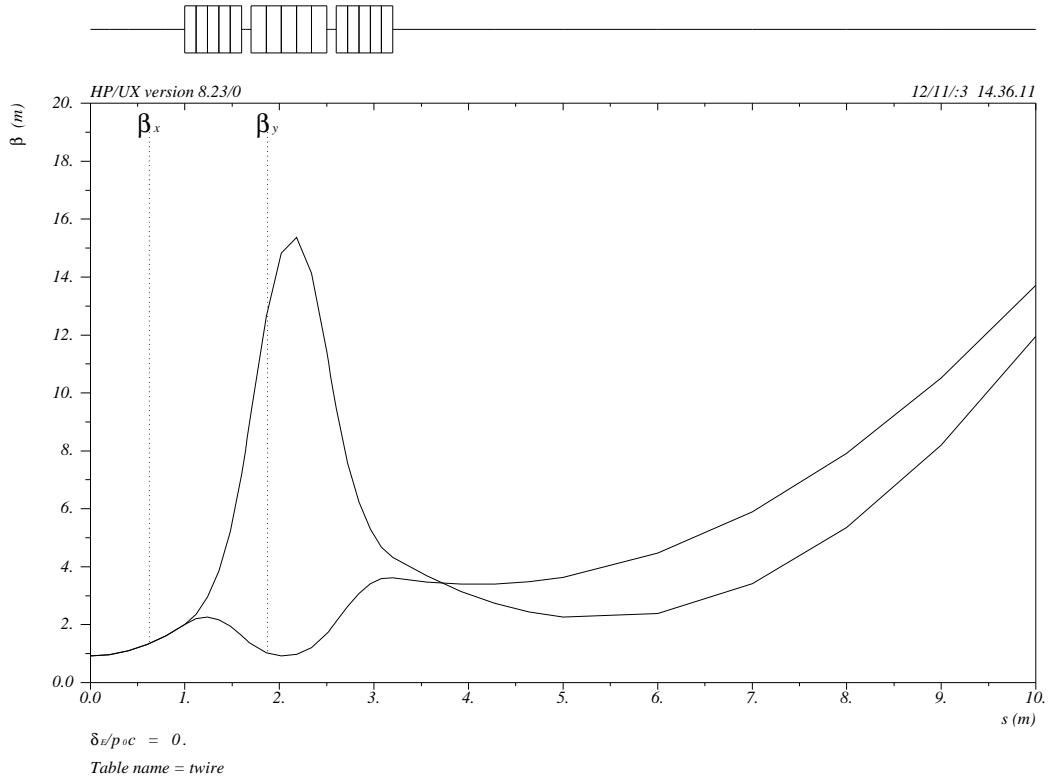

Figure 12: Electron IR lattice for the electron-ion collider eRHIC. 


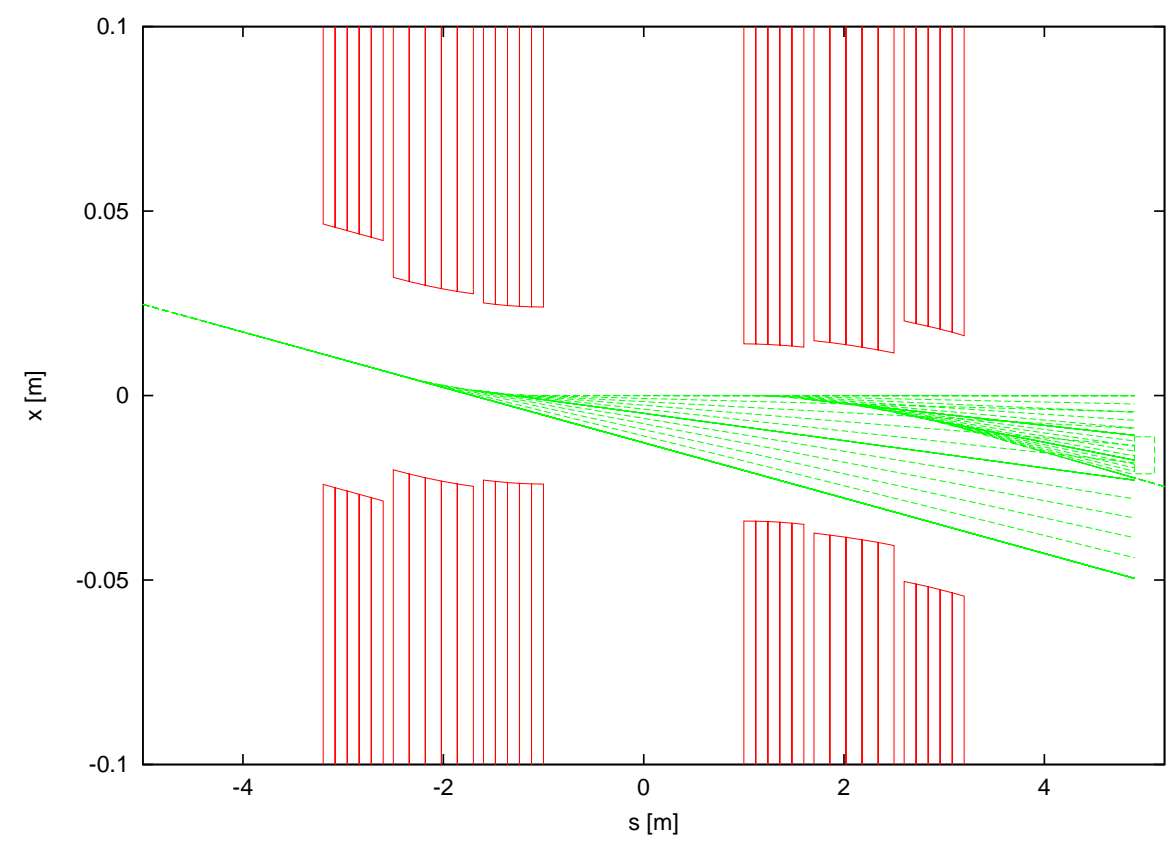

Figure 13: IR geometry and synchrotron radiation fan. The electron beam enters the IR from the left. The septum of the Q1 magnet is indicated by a box on the right side. 